\section{Referenzinstitut für Bioanalytik}

\author{
A. M. Gressner ${ }^{1}$ und O. A. Gressner ${ }^{2}$ \\ ${ }^{1}$ Labor Dr. Wisplinghoff Berlin, Berlin, Deutschland \\ ${ }^{2}$ Labor Dr. Wisplinghoff Köln, Köln, Deutschland
}

\section{Synonym(e) RfB}

Definition Das RfB ist Referenzinstitution im Sinne der jeweils geltenden Richtlinien der Bundesärztekammer zur Qualitätssicherung im medizinischen Laboratorium und wird getragen von der Deutschen Gesellschaft für Klinische Chemie und Laboratoriumsmedizin e.V. (DGKL; > Deutsche Gesellschaft für Klinische Chemie und Laboratoriumsmedizin e.V. (DGKL)).

Beschreibung Das RfB entwickelt Verfahren zur internen und externen Qualitätssicherung ( $\triangleright$ Ringversuch) labordiagnostischer Methoden und führt in Zusammenarbeit mit den von der - Bundesärztekammer (BÄK) ernannten Ringversuchsleitern Ringversuche zur externen Qualitätssicherung durch. Als Referenzinstitution arbeitet das RfB nach den jeweils gültigen Richtlinien der BÄK zur Qualitätssicherung im medizinischen Laboratorium. Zu den Aufgaben gehört weiterhin die Entwicklung von $\triangleright$ Referenzmessverfahren (Referenzmethoden) und Referenzmaterialien ( $\triangleright$ Referenzmaterial). Es ist in inter- nationale Standardisierungsaktivitäten eingebunden und wird durch Mitglieder seines wissenschaftlichen Beirats in zahlreichen nationalen und internationalen Gremien, z. B. IFCC $(\triangleright$ International Federation of Clinical Chemistry and Laboratory Medicine), DIN/CEN/ISO vertreten. Die Geschäftsstelle in Bonn ist zuständig für die Organisation von Ringversuchen, wie Auswahl und Prüfung geeigneter Kontrollmaterialien, Ermittlung von Zielwerten, Verwaltung von Teilnehmerbestellungen, Probenversand sowie Präsentation und Kommentierung der Ringversuchsergebnisse.

\section{Adresse der Geschäftsstelle:}

DGKL-Referenzinstitut für Bioanalytik

Friesdorferstr. 153

D-53175 Bonn

Tel.: 02289268950

Fax: 022892689529

E-Mail: info@dgkl-rfb.de

Internet: www.dgkl-rfb.de

\section{Literatur}

Mitteilungen der Bundesärztekammer (2014) Neufassung der Richtlinien der Bundesärztekammer zur Qualitätssicherung laboratoriumsmedizinischer Untersuchungen. Dtsch Ärztebl 111(38):A1583-A1618 www.dgkl-rfb.de 